\section{Niveles de hemoglobina glicosilada en pacientes con periodontitis crónica}

\author{
Glycated hemoglobin levels in patients with chronic periodontitis
}

\begin{abstract}
Resumen
Objetivo: Evaluar si existe relación entre el nivel de hemoglobina glicosilada y la presencia de periodontitis crónica. Materiales y método: se evaluaron 77 pacientes, agrupados en función a si presentaban o no periodontitis crónica. Los datos fueron recolectados en una ficha donde se consideró edad, género, índice de masa corporal (IMC) y antecedentes de dislipidemias, se llenaron los periodontogramas para establecer al grupo de pertenencia de los pacientes, se les envió a laboratorio clínico para recabar y procesar las pruebas de hemoglobina glicosilada. Todos firmaron el consentimiento informado. Resultado: se obtuvo un $5,58 \%$ de HbAlc para el grupo con periodontitis crónica (38 pacientes), y un 5,31\% para el grupo sin periodontitis (39 pacientes), valores sin ajustar, se determinó una diferencia significativa $(P=0,000)$, al realizar el estudio de las variables de riesgo, se obtuvo que no hay influencia estadística del género ni del IMC. Considerando estos factores la diferencia sigue siendo significativa $(\mathrm{P}=0,001)$. No se halló diferencia significativa $(\mathrm{P}=0,921)$ entre varones $(5,58 \%)$ y mujeres $(5,60 \%)$ con periodontitis crónica. Tampoco hubo diferencia significativa $(\mathrm{P}=0,230)$ entre varones $(5,33 \%)$ y mujeres $(5,29 \%)$ sin periodontitis crónica. Conclusiones: existe diferencia significativa en el porcentaje de hemoglobina glicosilada y la presencia de periodontitis crónica. No existe diferencia significativa en los pacientes en función al género, en los grupos con y sin periodontitis crónica. El análisis de factores de riesgo, mostró que los niveles elevados de Hemoglobina Glicosilada se deben a periodontitis crónica, edad del paciente y presencia de dislipidemias, más no del género y el IMC.
\end{abstract}

Palabras clave: periodontitis crónica, hemoglobina A glucosilada, análisis, diagnóstico.

\begin{abstract}
Objective: To evaluate the correlation between the level of glycosylated hemoglobin and the presence of chronic periodontitis. Materials and method: 77 patients, were grouped according to whether or not chronic periodontitis. Data were collected where took it age, gender, body mass index (BMI) and a history of dyslipidemia, periodontal chart were written to set membership group of patients, they were sent to clinical laboratory to collect and process glycosylated hemoglobin samples. All signed informed consent. Results: $\mathrm{HbA}_{1 \mathrm{c}}$ was $5.58 \%$ for chronic periodontitis group, and $5.31 \%$ for no periodontitis group, unadjusted values, determined it there was statistically significant difference $(P=0.000)$. Taking the risk variables it was obtained no statistical influence of gender or BMI. Even considering these risk factors, it remained significant difference $(P=0.001)$. No significant differences were found $(P=0.921)$ among men (5.58\%) and women (5.60\%) with chronic periodontitis. In the group of patients without chronic periodontitis there is no significant difference $(\mathrm{P}=0.230)$ between males $(5.33 \%)$ and women (5.29\%). Conclusions: A statistically significant difference was found in the percentage of Hemoglobin and the presence of chronic periodontitis. No significant difference was found in patients according to gender, both in the group with chronic periodontitis, such as those without chronic periodontitis. The analysis of risk factors showed that the causes of the high levels of percentage of Hemoglobin were the presence of chronic periodontitis, patient age and the presence of dyslipidemia, but not gender and Body Mass Index (BMI).
\end{abstract}

Keywords: chronic periodontitis, hemoglobin A glycosylated ,analysis, diagnosis.

\section{Introducción}

La enfermedad periodontal es una de las enfermedades orales más prevalentes, después de la caries dental, es una enfermedad infecciosa, producto de la inflamación de tejidos de soporte dentario, disminución de soporte óseo y pérdida progresiva de adherencia clínica gingival. Su etiología es multifactorial, tanto por factores microbiológicos, como predisposición del hués- ped $^{1}$. Usualmente es de evolución lenta, aunque en presencia de ciertos agentes ambientales o sistémicos negativos, se puede acortar el tiempo de aparición, además de alterar negativamente la respuesta del hospedero ante la acumulación de placa bacteriana, asimismo existen otros factores que alteran también la severidad y grado de la enfermedad como son enfermedades cardiovasculares, tabaquismo y diabetes ${ }^{2}$.

\section{Victor Eder Velásquez Machuca', Andrew Alejandro Estrada², Sofía Espinoza Escajadillo², Sixto García Linares ${ }^{3}$.}

1. Facultad de Odontología, UNMSM.

2. Departamento de Ciencias Básicas, Facultad de Odontología, U.N.M.S.M.

3. Departamento de Periodoncia, Facultad de Odontología, U.N.M.S.M.

Correspondencia:

Sixto Garcia Linares sgarcial@unmsm.edu.pe

Facultad de Odontología de la Universidad Nacional Mayor de San Marcos.

Av. Germán Amézaga 375, Lima 1, Perú

Coautores

Andrew Alejandro Estrada

alesandrew@gmail.com

Sofía Espinoza Escajadillo

sofiabelinda@hotmail.com
Fecha de Recepción:

Fecha de Aceptación:

Alrededor de 382 millones de personas en el mundo viven con diabetes mellitus tipo 2, lo cual representa una importante inversión económica en el manejo y cuidado de pacientes con esta enfermedad, aun mas cuando el $80 \%$ de estas personas viven en países con bajos y medianos ingresos. La mortalidad por las complicaciones de esta enfermedad es de alrededor 5.1 millones al ańo, y casi la mitad de estas en menores de 60 años. Se define como una 
situación clínica en la cual el organismo no puede producir suficiente hormona de insulina o esta no puede ser utilizada efectivamente por el organismo, esta hormona permite que las células del organismo puedan tomarla y usarla como energía del metabolismo ${ }^{3}$.

Desde el 2009, la ADA (American Diabetes Association) ha establecido protocolos para sistematizar el uso de la prueba de laboratorio de Hemoglobina Glicosilada, para el diagnóstico y control de la glucemia de pacientes en riesgo de padecer y en quienes ya se encuentra establecida esta enfermedad ${ }^{4}$. Existen estudios epidemiológicos que relacionan una mayor prevalencia de enfermedad periodontal en pacientes con diabetes mellitus tipo $2^{5}$, así como también hay otros en los que los que pacientes que presentan un mal control glucémico, tienen un mayor grado de severidad de la enfermedad periodon$\mathrm{tal}^{6}$. Existen diversos estudios en los que señalan que la enfermedad periodontal está relacionada a un pobre control glucémico, aun cuando los mecanismos fisiopatológicos no quedan del todo claros. $^{7,8}$

Existen diversos estudios donde comparan los niveles de hemoglobina glicosilada en pacientes sin diabetes, pero con periodontitis crónica y controles sanos. Evalúan criterios periodontales, como sangrado al sondaje, profundidad de bolsa y pérdida de adherencia clínica para determinar la presencia de periodontitis crónica. Encuentran diferencias significativas entre ambos grupos, así como también, que esta diferencia aumenta en función a la edad y si el paciente es fumador. ${ }^{9,10}$ Debido a la ausencia de información nacional sobre la relación de una enfermedad odontológica prevalente y una enfermedad crónica degenerativa, es que se plantea realizar una investigación tomando estas dos enfermedades de alta prevalencia.

\section{Materiales y método}

Los pacientes fueron seleccionados en el área de periodoncia del Hospital Centro Médico Naval -CMST. El comité de ética de dicho hospital evaluó y aprobó el estudio, y todos los pacientes proporcionaron su consentimiento informado por escrito.

Los pacientes tenían entre 30 y 65 años de edad, fueron criterios de inclusión que no presenten diagnóstico de diabetes mellitus, con 15 piezas dentarias como mínimo, y no tengan registro de prescripción antibiótica mayor a 14 días durante los últimos 6 meses. En el Servicio de Laboratorio Clínico, se invalida el test cuando se presentan antecedentes de anemias, eritrocemias $\mathrm{u}$ homocigosis, embarazo o reciente perdida sanguínea reciente en cantidad considerable, se excluyen pacientes con esas condiciones.

Los pacientes fueron seleccionados en función a la revisión clínica, para ello se utilizó una sonda periodontal calibrada por la OMS (Maillefer, 11B). Las medidas clínicas consideradas fueron: sangrado al sondaje, evaluado en los 6 sitios de todos los dientes. Los casos de periodontitis crónica fueron definidos con por lo menos $\geq 2$ sitios interproximales con pérdida de adherencia $\geq 3 \mathrm{~mm}$ y $\geq 2$ sitios interproximales con profundidad al sondaje $\geq 4 \mathrm{~mm}$, sangrado al sondaje y presencia de placa mineralizada. Los pacientes sanos no tenían una profundidad de sondaje mayor a 4 $\mathrm{mm}$, sangrado al sondaje en menos del $15 \%$ de sitios dentales y sin tratamiento periodontal anterior en los últimos 6 meses. Todo se registró en los periodontogramas de las historias clínicas del hospital.

Las covariables de edad y género, fueron recogidas vía cuestionario. El índice de masa corporal (IMC) se obtuvo en el momento en el Área de Evaluación Psicofísica del Hospital, y las dislipidemias, en el registro de laboratorio clínico del nosocomio en mención. $\mathrm{HbA}_{1 \mathrm{c}}$ fue medida en el Servicio de Laboratorio Clínico mediante el Kit de reactivos de HbA1c "DIMENSION", según el principio de Inmuno Ensayo de Inhibición Turbidimétrico, certificados por la National Hemoglobin Standardization Program.

\section{Resultados}

Se examinaron 77 pacientes que estuvieron de acuerdo a participar en el estudio, firmaron el consentimiento informado, y cumplían con los criterios de inclusión. De los cuales 64 (83,1\%) eran del género masculino y $13(16,9 \%)$ del género femenino.

Se encontró $5,58 \%$ como media de HbA1c en el grupo de pacientes con periodontitis crónica y 5,31\% para el grupo sin periodontitis crónica, estos valores con las variables sin ajustar, es decir sin considerar los factores de riesgo analizados. Esta diferencia fue estadísticamente significativa $(\mathrm{P}=0,000)$.

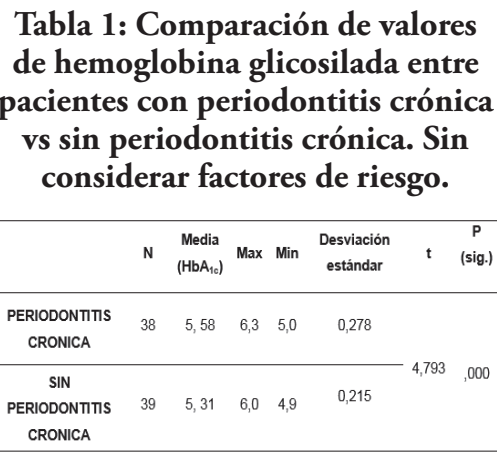

Para el análisis estadístico considerando los factores de riesgo, es decir tomando aquellos factores que puedan alterar los valores de $\mathrm{HbA}_{1 c}$, se eliminan aquellos pacientes valores fuera del rango de normalidad según el factor a analizar. Se encontró $5,61 \%$ de HbA1c para el grupo con periodontitis crónica, y $5,30 \%$ para el grupo sin periodontitis crónica. La diferencia de estos valores también fue estadísticamente significativa $(\mathrm{P}=0,001)$.

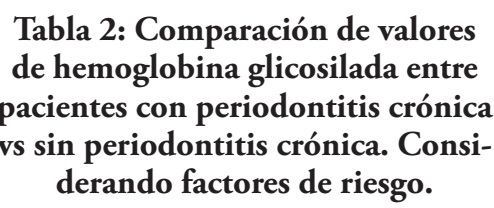

\begin{tabular}{|lccccc|}
\hline & N & $\begin{array}{c}\text { Media } \\
\text { (HDA1C) }\end{array}$ & $\begin{array}{c}\text { Desviación } \\
\text { estándar }\end{array}$ & $t$ & $P$ (sig.) \\
\hline CON PERIODONTITS & 17 & 5,61 &, 322 & & \\
\hline SIN PERIODONTTIS & 26 & 5,30 &, 229 & & \\
& & & & & \\
\hline
\end{tabular}

Cuando se realizó el análisis de regresión logística no lineal para determinar el grado de afectación estadística de cada factor de riesgo (variable interviniente), se encontró que la presencia de Periodontitis Crónica si tiene afectación estadística sobre la HbA1c $(\mathrm{P}=0,000$; Beta=0,37). La edad del paciente también tenía una correlación estadística $(\mathrm{P}=0,000 ;$ Beta=0,48). Y la presencia del factor de presencia de dislipidemias también tenía dicha correlación $(\mathrm{P}=0,020$, Beta $=0,28)$. Las variables de género del paciente $(\mathrm{P}=0,604)$ e Índice de Masa Corporal $(\mathrm{P}=0,068)$ no presentaron significancia estadística sobre los valores de $\mathrm{HbA}_{1 \mathrm{c}}$. 
Tabla 3: Regresión logística no lineal de factores de riesgo para niveles elevados de Hemoglobina Glicosilada.

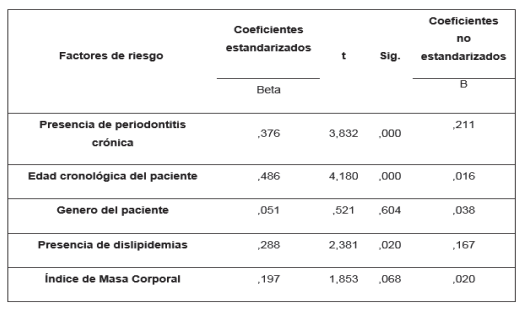

$\mathrm{Al}$ analizar los valores de $\mathrm{HbA}_{1 \mathrm{c}}$ en función al género del paciente en el grupo con presencia de periodontitis crónica, se halló una media de 5,58\% para el género masculino, y 5,60\% para el femenino, esta diferencia no tuvo significancia estadística $(\mathrm{P}=0,921)$.

Tabla 4: Comparación de hemoglobina glicosilada en pacientes con periodontitis crónica según el género.

\begin{tabular}{lccccc}
\hline $\begin{array}{c}\text { PERIODONTITIS } \\
\text { CRONICA }\end{array}$ & $\mathrm{N}$ & $\begin{array}{c}\text { Media } \\
\text { (HbA1c) }\end{array}$ & $\begin{array}{c}\text { Desviación } \\
\text { estándar }\end{array}$ & $\mathrm{t}$ & $\mathrm{P}$ (sig.) \\
\cline { 1 - 4 } Masculino & 32 & 5,58 &, 264 & &, 921 \\
\cline { 1 - 4 } Femenino & 6 & 5,60 &, 374 & & \\
\hline
\end{tabular}

Los valores de HbA1c, según el género en el grupo que no presentaba periodontitis crónica, se tuvo una media de $5,33 \%$ para el género masculino, y $5,29 \%$ en el femenino, dicha diferencia tampoco fue estadísticamente significativa $(\mathrm{P}=0,230)$.

Tabla 5: Comparación de hemoglobina glicosilada en pacientes sin periodontitis crónica según el género.

\begin{tabular}{cccccc}
\hline $\begin{array}{c}\text { SIN PERIODONTITIS } \\
\text { CRONICA }\end{array}$ & $\mathrm{N}$ & $\begin{array}{c}\text { Media } \\
\text { (HbA1c) }\end{array}$ & $\begin{array}{c}\text { Desviación } \\
\text { estándar }\end{array}$ & $\mathrm{t}$ & $\mathrm{P}$ (sig.) \\
\hline Masculino & 32 & 5,33 &, 221 & & \\
\hline Femenino & 7 & 5,29 &, 170 & & \\
\hline
\end{tabular}

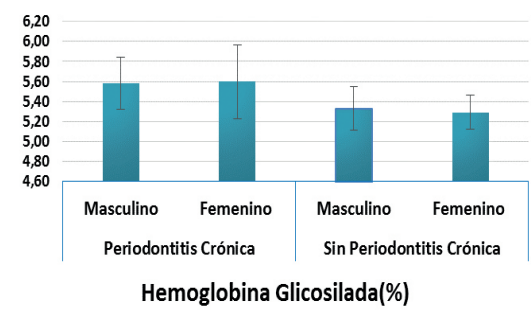

Figura 1: Porcentaje de Hemoglobina Glicosilada en pacientes con y sin periodontitis crónica según género.

\section{Discusión}

Se halló diferencia estadísticamente significativa del porcentaje de Hemoglobina Glicosilada $\left(\mathrm{HbA}_{1 c}\right)$ según la presencia o ausencia de Periodontitis Crónica, en ambos casos, con y sin ajustes de los factores de riesgo. Estos resultados concuerdan con los de Wolff R. ${ }^{10}$ y Rajan P. ${ }^{9}$ quienes obtienen también obtienen diferencias significativas, cuando realizan los ajustes de factores de riesgo, sin embargo, ellos, antes de dicho ajuste estadístico no obtienen diferencia alguna. Los resultados se contraponen a los de Saxena R. ${ }^{11}$ quien no refiere diferencias estadísticas entre los grupos con y sin periodontitis crónica.

En este estudio no se halló diferencia significativa intra grupo en función al género, aunque son coincidentes con Muñera M..$^{12}$ y Selvin E. ${ }^{13}$, se debe tener cuidado en su interpretación, puesto que aun la estadística considere que no existe diferencia significativa, habría sido de mayor relevancia en la medida que se haya podido acceder a un grupo más amplio de pacientes de género femenino, puesto que solo se contó con 13 y en su mayoría fueron varones (64). Esta diferencia se debe al lugar donde se realizó el estudio, al ser una institución miliar, hay una mayor cantidad de personal de género masculino que femenino. (Explicar a qué se debió esta desigualdad en el número entre hombres y mujeres.)

En función a la edad, se presenta como un factor de riesgo importante, coincide con los resultados de Rajan P. ${ }^{9}$

La presencia de dislipidemias, concentraciones anormales de lipoproteínas sanguíneas. También se considera un factor de riesgo para diabetes, aunque no hay antecedentes en los que consideren este factor como predisponente a periodontitis crónica, estudios adicionales que consideran que la presencia de dislipidemias está asociada a un mayor riesgo de presentar enfermedades crónicas degenerativas como la diabetes mellitus, como señala Canalizo M. ${ }^{14}$

El índice de masa corporal no representa un factor de riesgo estadísticamente significativo, similar resultado obtuvo Saxena R. ${ }^{11}$

\section{Conclusiones}

1. Se encontró diferencia estadísticamente significativa en el porcentaje de Hemoglobina Glicosilada entre pacientes con y sin de Periodontitis Crónica

2. No se halló diferencia significativa entre la Hemoglobina Glicosilada de los pacientes en función al género, tanto en el grupo con periodontitis crónica, como aquellos que no presentaban periodontitis crónica.

3. Se halló como factores de riesgo de niveles elevados en el porcentaje de hemoglobina glicosilada a la periodontitis crónica, edad del paciente y las dislipidemias, más no son factores de riesgo el género y el Índice de Masa Corporal (IMC).

\section{Referencias Bibliográficas}

1. Lindhe J, Ranney R, Lamster. I. Consensus Report: Chronic Periodontitis. Ann Periodontol. 1999; 4(1): 38 .

2. Flemmig T. Periodontitis. Ann Periodontol. 1999; 4:32-37.

3. International Diabetes Federation. IDF Diabetes Atlas. 6th ed. IDF. Brussels: 2013. Pag 13- 27.

4. Nathan D. International Expert Committee Report on the Role of the A1C Assay in the Diagnosis. Diabetes Care. 2009; 32(7): 13271334.

5. Sanz I, Bascones A. Diabetes mellitus: Su implicación en la patología oral y periodontal. Avances en Odontoestomatología. 2009; 25 (5): 249-263.

6. Taylor GW. Bidirectional interrelationships between diabetes, and periodontal diseases: an epidemiologic perspective. Annals of Periodontology 2001. (6):99-112.

7. Méndez J. Productos finales de glicación avanzada y complicaciones crónicas de la diabetes mellitus. Gac Méd Méx. 2003; 39 (1):4955.

8. Dyer D, Dunn J, Thorpe S, Bailie K, Lions T, Maccande C, Baynes J. accumulation of Maillard reaction products in skin collagen in diabetes and agging. J Clin Invest. 1993; 91: $2463-2469$.

9. Rajan P, Nera M, Kumar A, Medandrao N, Chetan S. Comparison of glycosylated hemoglobin $\left(\mathrm{HbA}_{1 \mathrm{c}}\right)$ levels in patients with chronic periodontitis and healthy 
controls. Dent Res J. 2013. 10(3): 389-393.

10. Wolff R, Wolff L, Michalowicz B. A pilot study of glycosylated hemoglobin levels in periodontitis cases and healthy controls. J Periodontol. 2009; 80(7):1057-1061.

11. Saxena R, Deepika P. Comparison of glycosylated hemoglobin levels in periodontitis patients and healthy controls: A pilot study in
Indian population. Indian J Dent Res. 2012; 23(3):368 - 372.

12. Múnera M, Restrepo M., Gómez L., Mesa D., Ramirez B. Hemoglobina glicosilada $A_{1 c}$ vs glucemia plasmática en ayunas de pacientes ambulatorios de un laboratorio médico. Rev. Salud Pública. 2011; 13 (6): 980-989

13. Selvin E, Zhu H, Brancati FL. Elevated A1c in adults without a history of diabetes in the U.S. Diabetes Care 2009; 32(5): 828-833.

14. Canalizo M, Favela E, Salas J, Gómez R, Jara R, Torres L, Viniegra A. Diagnóstico y tratamiento de las dislipidemias. Rev. Med. Inst. Mex. Seguro Soc. 2013. 51(6): 700-709. 